\title{
Cognitive functioning at the age of 10 years among children born extremely preterm: a latent profile approach
}

\author{
Timothy Heeren ${ }^{1}$, Robert M. Joseph ${ }^{2}$, Elizabeth N. Allred ${ }^{3}$, Thomas M. O'Shea ${ }^{4}$, Alan Leviton ${ }^{3}$ and Karl C.K. Kuban ${ }^{5}$
}

BACKGROUND: School-age children born extremely preterm (EP) are more likely than their term peers to have multiple neurocognitive limitations. We identify subgroups of EP children who share similar profiles on measures of intelligence quotient (IQ) and executive function (EF), and describe the nature and prevalence of cognitive impairment in EP children. METHODS: On the basis of measures of $\mathrm{IQ}$ and EF, subgroups of EP children with common neurocognitive function are identified using latent profile analysis (LPA). On the basis of these subgroups, we describe the nature and prevalence of impairment in EP children, and examine associations between cognitive function, gestational age, and academic achievement. Classification of neurocognitive function using $\mathrm{IQ}$ and EF is compared with a standard classification based on IQ Z-scores.

RESULTS: LPA identified four neurocognitive profiles in EP children, with $34 \%$ of EP children classified as normal, $41 \%$ low-normal, 17\% moderately impaired, and 8\% severely impaired. Impaired children exhibited global impairment across cognitive domains, whereas children in the lownormal group tended to have impaired inhibition relative to their reasoning and working memory skills.

CONCLUSION: Within categories of EP children defined in terms of $I Q$, there is substantial variation in EF; thus, both $I Q$ and EF assessments are needed when describing school-age outcome of EP children.

D espite reductions in mortality and major medical morbidities among children born extremely preterm (EP) (1), EP children continue to be at significant risk for moderate-to-severe neurocognitive impairment that persists through the school years into young adulthood (2). As a consequence of these impairments, children born preterm are at an increased risk of poor school performance (3-5). Estimates of the prevalence of intellectual impairment, defined as a total intelligence quotient (IQ) below $70(<-2$ $Z$-scores below the mean), have ranged from $45 \%$ in a cohort of 11-year-olds born before 26 weeks (6) to $15 \%$ in a cohort of 16-year-olds with birth weight $<1,250 \mathrm{~g}$ and a mean gestational age of 28 weeks (7). Among children with subnormal-but not impaired-IQ, overall function is likely determined by IQ and other neurocognitive abilities, especially executive function $(8,9)$. Gaining a better understanding of the etiology of neurocognitive impairment and the antenatal and neonatal antecedents of unfavorable cognitive outcomes among EP children is a primary aim of epidemiological research on prematurity.

Existing studies of cognitive outcomes among individuals born EP illustrate several challenges to formulating an operational definition of cognitive impairment. Often, children are categorized as cognitively impaired based on IQ $<70$ (more than 2 SDs below the normative mean), both in epidemiological studies $(6,7)$ and clinical trials $(10,11)$. However, children born preterm exhibit impairment in virtually all domains of cognitive function (2), and cognitive deficits in EP children tend to co-occur $(5,12,13)$. Evaluating each measurement domain for associations with antecedent factors or later outcomes results in multiple significance tests and difficulties in parceling the effects of highly correlated measures. Alternatively, summarizing a child's impairment based on their maximum impairment level across a set of measures or domains (6) fails to describe either the breadth of impairment or differences in patterns of impairment across measures. In fact, a recent systematic review found that no studies of risk factors for cognitive impairment among children born very preterm incorporated assessments of executive function into their measurements of outcome (14).

Latent profile analysis (LPA) operates by empirically identifying subgroups of children who share similar profiles on a set of measures. In this study, using LPA, we examine the nature and prevalence of cognitive functioning in EP children taking into account verbal and nonverbal IQ and executive functions. As a means of demonstrating content validity for this approach, we examine the association between cognitive profiles based on both IQ and EP at 10 years and lower gestational age, a risk factor for poorer cognitive outcomes $(6,15)$. We also compare cognitive profiles based on IQ and EF with a standard classification based on IQ Z-scores as

${ }^{1}$ Department of Biostatistics, Boston University School of Public Health, Boston, Massachusetts, USA; ${ }^{2}$ Department of Anatomy and Neurobiology, Boston University School of Medicine, Boston, Massachusetts, USA; ${ }^{3}$ Department of Neurology, Boston Children's Hospital, Boston, Massachusetts, USA; ${ }^{4}$ Department of Pediatrics, University of North Carolina School of Medicine, Chapel Hill, North Carolina, USA; ${ }^{5}$ Department of Pediatrics, Boston Medical Center, Boston, Massachusetts, USA. Correspondence: Timothy Heeren (tch@bu.edu) 


\section{Cognitive functioning in EP children $\quad$ Articles}

predictors of academic achievement and the need for special education.

\section{METHODS}

\section{Participants}

The ELGAN (Extremely Low Gestational Age Newborn) Study is a multicenter observational study of the risk of structural and functional neurologic disorders in EP children (16-18). During the years 2002-2004, women delivering before 28 weeks' gestation in 11 cities in 5 states were asked to enroll in the study. Overall, 1,249 mothers of 1,506 infants consented to participate. Of 1,200 EP infants who survived to the age of 2 years, the parents of 1,102 (92\%) consented to have their child participate in follow-up of developmental outcomes at the age of 2 years. Families of children with data on inflammatory biomarkers at birth $(n=966)$, most of whom participated in the age 2 follow-up, were invited by mail and then phone to participate in the age 10 follow-up. Families of 889 of the 966 (92\%) targeted children participated in the age 10 study. For 11 families, the parents participated in the follow-up interview but the child did not participate in the follow-up evaluation, and an additional 5 children were unable to undergo neurocognitive assessment at the visit. The remaining 873 children participated in the neurocognitive evaluation and are included in these analyses. Enrollment and consent procedures for this follow-up study were approved by the institutional review boards of all participating institutions.

\section{Measures}

Assessments were selected to provide a broad overview of neurocognitive and academic function given the time constraints. This report focuses on two key indices of cognitive ability: general cognitive ability, or IQ, and executive function.

Verbal and nonverbal reasoning. General cognitive ability (or IQ) was assessed with the School-Age Differential Ability Scales-II (DASII) Verbal and Nonverbal Reasoning Scales. The DAS has several advantages for characterizing the wide range of cognitive ability in an EP sample, including more sensitive basal items than the Wechsler and other IQ scales, and extended standard scores (down to 31) for lower-ability individuals. In addition, the DAS nonverbal reasoning subtests require minimal visual-spatial processing and fine-motor dexterity allowing more accurate estimate of nonverbal reasoning in preterm children.

Executive function. Two subtests from the DAS-II and five subtests from the NEPSY-II were used to assess executive function. The DAS-II Recall of Digits Backward and Recall of Sequential Order subtests measured working memory. From the NEPSY-II, the Auditory Attention and Response Set subtests measured sustained attention, set-shifting, and inhibition; the Inhibition-Inhibition and Inhibition-Switching subtests measured inhibition and set-shifting; and the Animal Sorting subtest measured concept generation and mental flexibility.

Academic achievement. The Wechsler Individual Achievement Test-III (WIAT-III) Word Reading and Numerical Operations subtests were used to assess children's basic reading and math skills. Whether a child had an individual education plan (IEP), repeated a grade, or was in a class for children with special needs was reported by the parent.

We defined impairment on any individual cognitive or academic achievement measure as a score two or more SDs below the normative mean.

\section{Statistical Methods}

LPA was used to identify subgroups of EP children with similar profiles of scores on nine variables measuring verbal and nonverbal IQ (DAS-II Verbal and Nonverbal Reasoning Scales), working memory (DAS-II Recall of Digits Backwards, Recall Sequential Order), concept generation and mental flexibility (NEPSY-II Animal Sorting), auditory attention and set switching (NEPSY-II Auditory Attention, Response Set), and simple inhibition and inhibition shifting (NEPSY-II Inhibition-Inhibition and Inhibition-Switching). LPA was conducted using Mplus 7.11 (19), which provides maximum likelihood estimation and includes children with missing data on some measures under the missing at random assumption. Successive LPA models were fit to the data, starting with a model including only one profile and increasing up to six profiles. To determine the optimal number of profiles, the fit of each model was examined through the Bayesian Information Criteria (BIC) (20), the sample-size-adjusted Bayesian Information Criteria (SSABIC) (21) and the Lo-Mendell-Rubin-adjusted likelihood ratio test (LMR) (22). For both the BIC and SSABIC, lower values indicate better fit; for the LMR, a significant result indicates that the model is a better fit than the model with one less profile. Entropy values were also used to determine the model with the optimal LPA solution. Entropy values range from 0 to 1 . The closer the value is to 1 , the better the separation of the profiles (23).

Children were categorized by their most likely latent profile for further analysis. A concern with categorizing based on the most likely profile is that it fails to account for potential misclassification (24). However, the impact of misclassification is small when entropy is high $(>0.80)$.

Children are also categorized using a standard classification based on IQ alone. DAS verbal and nonverbal IQ scores were converted to $Z$-scores (giving the IQ as SDs from the mean, by subtracting the normative mean of 100 and dividing by the normative SD of 15) and then averaged. We refer to this averaged IQ $Z$-score as $Z_{\mathrm{IQ}}$. Children are classified as having severely impaired, moderately impaired, or nonimpaired IQ if their $Z_{\mathrm{IQ}}$ is -2 or below, between -2 and -1 , or above -1 .

Prevalences of the cognitive profiles are reported for the overall sample and by gestational age (categorized as 23-24, 25-26, and 27 weeks), with $95 \%$ confidence intervals (CI); the difference in the prevalences of cognitive profiles by gestational age was tested using the $\chi^{2}$ test. Analysis of variance followed by Scheffe's procedure was used to test for a difference among profile groups based on both IQ and $\mathrm{EF}$ on mean academic achievement, and $\chi^{2}$ analysis was used to test for the difference among profiles on the percentage of children with an IEP, repeating a class, and in a special needs class. Comparison of academic achievement and educational needs across cognitive function profiles defined by LPA on both IQ and EF, for children with a specific $Z_{\mathrm{IQ}}$ categorization, were conducted through ANOVA and $\chi^{2}$.

Twenty-four children in the sample had severe motor impairment or functional blindness that could invalidate their cognitive assessment. These children were included in the main analyses. In secondary analyses, the LPA was repeated excluding these children.

\section{RESULTS}

\section{Sample Description}

Of the 873 participants, 17 (1.9\%) had severe motor impairment (Gross Motor Function Classification System =5) and 7 (0.8\%) had functional blindness ( 2 children had both severe motor impairment and functional blindness). Participants who were not able to obtain a basal score (because of cognitive impairment) on a given test were assigned a floor score for that test. Of children with severe motor or visual impairment, 17 did not achieve basal scores on any test and 2 obtained basal scores on some but not all measures. Of children without severe motor or visual impairment, 12 did not achieve basal scores on any test and 9 obtained basal scores on only some tests. In 


\section{Articles | Heeren et al.}

summary, a total of 29 children were assigned floor scores on all tests, and 11 were assigned floor scores on some tests.

Of the 873 children in this sample, $21 \%$ were born at 2324 weeks' gestation, $45 \%$ at $25-26$ weeks' gestation, and $34 \%$ at 27 weeks' gestation. Overall, $49 \%$ are girls. A total of $63 \%$ of mothers identified as white, $26 \%$ as black, and $10 \%$ as Hispanic. In all, $41 \%$ had a high school education or less, and $35 \%$ were eligible for government-provided medical care insurance at the time of her child's delivery.

\section{Cognitive Profiles Based on $\mathrm{Z}_{\mathrm{IQ}}$}

Sixty-six percent $(n=573)$ of the sample had IQ in the nonimpaired range with $Z_{\mathrm{IQ}}$ above $-1,19 \%$ had moderately impaired IQ with $Z_{\mathrm{IQ}}$ between -2 and -1 , and $15 \%$ had severely impaired IQ with $Z_{\mathrm{IQ}}$ of -2 or below.

\section{Cognitive Profiles from Latent Profile Analysis of IQ and EF}

On the basis of the fit indices, the four-profile model provides the best fit for the data, with substantial improvement over the three-profile model in the Akaike Information Criteria and Bayesian Information Criteria values, and no significant improvement in fit with a five-profile model based on the LMR test $(P=0.07)$. The four-profile model provides good separation of the subgroups with an entropy value of 0.88 .

Profiles and prevalence. The means and SDs of the cognitive measures used in the LPA are plotted as SDs about the normative means in Figure $\mathbf{1}$ and given for each profile in Supplementary Table S1 online. Overall, 34\% (95 CI\%: 31\%,

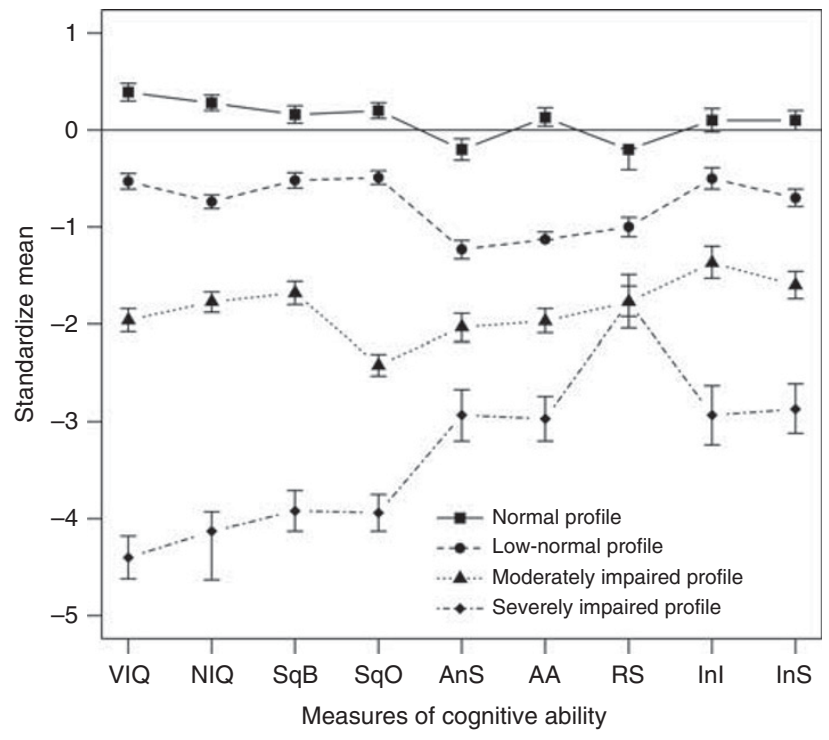

Figure 1. Means of cognitive ability measures (in SDs from the norm) for the four latent profile analysis (LPA) classes. Bars represent 95\% confidence interval $(\mathrm{Cl})$ around the mean. AA, NEPSY-II Auditory Attention; AnS, NEPSY-II Animal Sorting; Inl, NEPSY-II InhibitionInhibition; InS, NEPSY-II Inhibition-Switching; NIQ, DAS-II Nonverbal Reasoning; RS, NEPSY-II Response Set; SqB, DAS-II Recall of Digits Backward; SqO, DAS-II Recall of Sequential Order; VIQ, DAS-II Verbal Reasoning.
$38 \%$ ) of children had a "normal" cognitive functioning profile, which is characterized by mean scores within the normal range on all nine cognitive and executive function measures. Fortyone percent $(95 \% \mathrm{CI}: 38 \%, 45 \%)$ of children had cognitive function consistent with a "low-normal" profile. Mean values on the nine tests for this group ranged from $0.5 \mathrm{SD}$ below the norm (for IQ) to over 1 SD below the norm (for inhibition/setshifting). Seventeen percent (95\% CI: 14\%, 19\%) had a "moderately impaired" profile, with means on the nine measures between 1.5 and 2.5 SDs below normative values. Eight percent $(95 \% \mathrm{CI}: 6 \%, 10 \%)$ of children exhibited a "severely impaired" cognitive function profile, with means on the nine measures $\sim 3$ to 4 SDs below normative values.

Excluding the 24 children with severe visual and motor impairments yielded very similar results on the LPA, with entropy of 0.88 for the four-profile model and $P=0.418$ for the LMR test showing no significant improvement with a fiveprofile model. Overall, $99 \%$ of children without visual or motor impairment fell into the same impairment profile for the LPAs on the full and restricted samples. For children without visual or motor impairment, $35,42,17$, and $6 \%$ were classified as having a normal, low-normal, moderately impaired, and severely impaired profile, respectively.

General vs. specific impairment. The percentage of children with impairment ( 2 or more SDs below the norm) on one or more individual measures of IQ or EF varied prominently with profile assignment (Table 1). For example, 99\% (95\% CI: 98\%, 100\%) of those with the normal cognitive function profile and $96 \%$ (95\% CI: $94 \%, 98 \%)$ of those with the low-normal cognitive function profile had either no impairment or impairment in only one domain, whereas $65 \%$ (95\% CI: $57 \%, 73 \%$ ) of those with the moderate impairment and $100 \%$ (95\% CI: 95\%, 100\%) of those with the severe impairment profile had both IQ and EF impairments. Children with moderate and severe impairment profiles exhibited global impairment, with high levels of impairment on all IQ and executive function measures. Children with the low-normal profile tended to have a relatively specific impairment or sets of impairment, largely with respect to executive function. For example, few of these children showed impairment in IQ or working memory measures, whereas $22 \%$ (95\% CI: 18\%, 26\%) showed impairment in sustained attention, 23\% (95\% CI: 19\%, $27 \%$ ) in concept generation, and $46 \%$ (95\% CI: $41 \%, 51 \%)$ in inhibition.

Cognitive function profiles by gestational age. Gestational age ranged from 23 to 27 weeks in our sample. Children born at 23-24 weeks' gestational age were most likely to have the severely impaired profile and least likely to have the normal profile (Table 2).

Comparison of Cognitive Function Categorizations Based on IQ and $\mathrm{EF}$ vs. $\mathrm{Z}_{\mathrm{IQ}}$

Of the $66 \%$ of EP children with $Z_{\mathrm{IQ}}$ above -1 , roughly half (34\% of the overall sample) were categorized as having normal cognitive function based on IQ and EF, and half 
Table 1. Percentage of children with 1 or more impairment on measures of verbal and nonverbal IQ and executive function, by cognitive function profile based on $\mathrm{IQ}$ and $\mathrm{EF}$

\begin{tabular}{ccccc}
\hline & \multicolumn{4}{c}{ LPA profile based on IQ and EF } \\
\cline { 2 - 5 } & $\begin{array}{c}\text { Normal } \\
(n=299)\end{array}$ & $\begin{array}{c}\text { Low-normal } \\
(n=360)\end{array}$ & $\begin{array}{c}\text { Moderately } \\
\text { impaired }(n=145)\end{array}$ & $\begin{array}{c}\text { Severely } \\
\text { impaired } \\
(n=69)\end{array}$ \\
\hline $\begin{array}{l}\text { Z-scores } \leq-2 \\
\text { None }\end{array}$ & 82 & 28 & $<1$ & 0 \\
IQ only & $<1$ & 3 & 0 & 0 \\
EF only & 18 & 66 & 34 & 0 \\
$\begin{array}{l}\text { Both IQ } \\
\text { and EF }\end{array}$ & 0 & 4 & 65 & 100 \\
\hline EF, executive function; IQ, intelligence quotient; LPA, latent profile analysis.
\end{tabular}

Table 2. Percentage of children with each cognitive function profile based on IQ and EF, by gestational age

\begin{tabular}{lccccc}
\hline Gestational age & \multicolumn{5}{c}{ Cognitive function profile } \\
\cline { 2 - 6 } & $N$ & Normal & $\begin{array}{c}\text { Low- } \\
\text { normal }\end{array}$ & $\begin{array}{c}\text { Moderately } \\
\text { impaired }\end{array}$ & $\begin{array}{c}\text { Severely } \\
\text { impaired }\end{array}$ \\
\hline 23-24 weeks & 180 & 23 & 39 & 21 & 17 \\
$25-26$ weeks & 395 & 33 & 42 & 18 & 7 \\
27 weeks & 298 & 43 & 41 & 13 & 3 \\
\hline
\end{tabular}

Distribution of cognitive profiles significantly differs by gestational age, $x^{2} P<0.001$.

Table 3. Cognitive profile classification based on IQ and EF vs. based on IQ only.

\begin{tabular}{lccccc}
\hline $\begin{array}{l}\text { Classification } \\
\text { based on IQ } \\
\text { only }\end{array}$ & \multicolumn{5}{c}{ Profile based on IQ and EF } \\
\cline { 2 - 6 } & Normal & $\begin{array}{c}\text { Low- } \\
\text { normal }\end{array}$ & $\begin{array}{c}\text { Moderately } \\
\text { impaired }\end{array}$ & $\begin{array}{c}\text { Severely } \\
\text { impaired }\end{array}$ & Overall \\
\hline$Z_{\mathrm{IQ}}>-1$ & $n=298$ & $n=264$ & $n=11$ & $n=0$ & $n=573$ \\
& $34.1 \%$ & $30.2 \%$ & $1.9 \%$ & & $65.6 \%$ \\
$-2<Z_{\mathrm{IQ}} \leq-1$ & $n=1$ & $n=94$ & $n=71$ & $n=0$ & $n=166$ \\
& 0.6 & $10.8 \%$ & $8.1 \%$ & & $19 \%$ \\
& $n=0$ & $n=2$ & $n=63$ & $n=69$ & $n=134$ \\
$Z_{\mathrm{IQ}} \leq-2$ & & $0.2 \%$ & $7.2 \%$ & $7.9 \%$ & $15.4 \%$ \\
& & & & & \\
& $n=299$ & $n=360$ & $n=145$ & $n=69$ & $n=873$ \\
Overall & $34.2 \%$ & $41.2 \%$ & $16.6 \%$ & $7.9 \%$ & \\
\hline
\end{tabular}

$E F$, executive function; $I Q$, intelligence quotient.

Percentages are percent of the overall sample.

(30\% of the overall sample) as having low-normal function (Table 3). Similarly, children with $Z_{\mathrm{IQ}}$ between -2 and -1 were roughly evenly divided into the low-normal and moderately impaired profiles based on IQ and EF, and children with $Z_{\mathrm{IQ}}$ of -2 or below are roughly evenly divided between the moderately and severely impaired profiles based on IQ and EF.
Table 4. Mean and SD of WIAT Word Reading (top row in each cell) and Numerical Operations scores, by cognitive function profiles based on IQ and EF, and classification based on IQ only

\begin{tabular}{|c|c|c|c|c|}
\hline \multirow{2}{*}{$\begin{array}{l}\text { Classification } \\
\text { based on IQ } \\
\text { only }\end{array}$} & \multicolumn{4}{|c|}{ Profile based on IQ and EF } \\
\hline & Normal & Low-normal & $\begin{array}{l}\text { Moderately } \\
\text { impaired }\end{array}$ & $\begin{array}{l}\text { Severely } \\
\text { impaired }\end{array}$ \\
\hline \multirow[t]{3}{*}{ Overall $^{a}$} & $107.8(11.8)$ & $94.4(13.2)$ & $77.9(14.4)$ & $51.1(15.1)$ \\
\hline & $103.2(12.3)$ & $90.7(11.7)$ & $71.8(14.3)$ & $44.4(10)$ \\
\hline & $n=299$ & $n=360$ & $n=145$ & $n=69$ \\
\hline \multirow[t]{3}{*}{$Z_{\mathrm{IQ}}>-1^{\mathrm{b}}$} & $107.8(11.7)$ & $96.4(12.7)$ & $88.5(11.4)$ & \\
\hline & $103.2(12.3)$ & $92.7(11.5)$ & $87.5(13)$ & \\
\hline & $n=298$ & $n=264$ & $n=11$ & $n=0$ \\
\hline \multirow[t]{3}{*}{$-2<Z_{\mathrm{IQ}} \leq-1^{\mathrm{a}}$} & - & 89 (13.2) & $81(15.4)$ & \\
\hline & - & $85.7(10.4)$ & $74.4(13.8)$ & \\
\hline & $n=1$ & $n=94$ & $n=71$ & $n=0$ \\
\hline \multirow[t]{3}{*}{$Z_{1 Q} \leq-2^{a}$} & & - & $72.6(11.4)$ & $51.1(15.1)$ \\
\hline & & - & $66.1(12.1)$ & $44.4(10)$ \\
\hline & $n=0$ & $n=2$ & $n=63$ & $n=69$ \\
\hline \multicolumn{5}{|c|}{$\begin{array}{l}\text { EF, executive function; } I Q \text {, intelligence quotient. } \\
\text { a } P<0.001 \text { from ANOVA comparing means across the row, for both WIAT Word } \\
\text { Reading and Numerical Operations, all pairwise comparisons } P<0.001 \text { from } \\
\text { Scheffe's procedure. } \\
b P<0.001 \text { from ANOVA comparing LPA profiles of Normal and Low-Normal, for } \\
\text { both WIAT Word Reading and Numerical Operations. }\end{array}$} \\
\hline
\end{tabular}

Cognitive function profile and academic achievement. Children with the moderate and severely impaired profiles based on IQ and EF were more likely to have lower scores on academic achievement measures of literacy and math (Table 4). Children with the moderately impaired cognitive function profile had mean WIAT-III achievement scores 1.5-2 SDs below the normative level, whereas children with the severely impaired profile had mean WIAT-III achievement scores 3 SDs below the norms. The mean academic performance scores for children with the normal profile were at or slightly above normative levels of achievement, whereas the scores for the low-normal profile children were just below the norm.

Among EP children with $Z_{\mathrm{IQ}}$ between -2 and -1 , those with low-normal profiles based on IQ and EF had significantly higher WIAT-III word reading and numerical operations scores than those with moderately impaired profiles (Table 4). Similarly, among children with $Z_{\mathrm{IQ}}$ of -2 or below, those with moderately impaired profiles based on IQ and EF had significantly higher achievement scores than those with severely impaired profiles based on IQ and EF.

Educational needs. Children with poorer cognitive function based on IQ and EF had more special ageneeds at school (Table 5). The percentage of children with an IEP increased 
Table 5. Percentage of children who had an IEP, repeated a grade, or enrolled in a special class, by cognitive function profiles based on IQ and $\mathrm{EF}$, and on IQ only

\begin{tabular}{|c|c|c|c|c|}
\hline \multirow{2}{*}{$\begin{array}{l}\text { Classification } \\
\text { by IQ only }\end{array}$} & \multicolumn{4}{|c|}{ Classification by IQ and EF } \\
\hline & Normal & Low-normal & $\begin{array}{l}\text { Moderately } \\
\text { impaired }\end{array}$ & $\begin{array}{l}\text { Severely } \\
\text { impaired }\end{array}$ \\
\hline \multirow[t]{4}{*}{ Overall ${ }^{\mathrm{a}}$} & $83(28 \%)$ & $188(52 \%)$ & $128(88 \%)$ & $68(99 \%)$ \\
\hline & $22(7 \%)$ & $75(21 \%)$ & $56(39 \%)$ & $9(13 \%)$ \\
\hline & $10(3 \%)$ & $36(10 \%)$ & 71 (49\%) & $64(93 \%)$ \\
\hline & $n=299$ & $n=360$ & $n=145$ & $n=69$ \\
\hline \multirow[t]{4}{*}{$Z_{\mathrm{IQ}}>-1^{\mathrm{b}}$} & $83(28 \%)$ & $132(48 \%)$ & $8(64 \%)$ & $n=0$ \\
\hline & $22(7 \%)$ & $53(20 \%)$ & $3(27 \%)$ & \\
\hline & $10(3 \%)$ & $18(6 \%)$ & $4(27 \%)$ & \\
\hline & $n=298$ & $n=264$ & $n=11$ & \\
\hline \multirow[t]{4}{*}{$-2<Z_{\mathrm{IQ}} \leq-1^{\mathrm{a}}$} & $n=1$ & $55(64 \%)$ & 61 (83\%) & $n=0$ \\
\hline & & $22(24 \%)$ & $34(46 \%)$ & \\
\hline & & $18(20 \%)$ & $30(42 \%)$ & \\
\hline & & $n=94$ & $n=71$ & \\
\hline \multirow[t]{4}{*}{$Z_{\mathrm{IQ}}<-2^{\mathrm{C}}$} & $n=0$ & $n=2$ & $59(98 \%)$ & 68 (99\%) \\
\hline & & & $19(32 \%)$ & 9 (13\%) \\
\hline & & & $37(60 \%)$ & $64(93 \%)$ \\
\hline & & & $n=63$ & $n=69$ \\
\hline
\end{tabular}

$E F$, executive function; $I Q$, intelligence quotient; IEP, individual education plan.

${ }^{a} P<0.001$ comparing percentages across the row for IEP, repeating a grade, and enrolling in a special class via $x^{2}$ test.

${ }^{\mathrm{b}} P<0.01$ comparing percentages across the row for IEP, repeating a grade, and enrolling in a special class via $X^{2}$ test.

${ }^{c} P=0.921$ for IEP, $P=0.011$ for repeating a grade, $P<0.001$ for special class comparing percentages across the row via $x^{2}$ test.

from $28 \%$ among those with a normal profile to 52,88 , and 99\% among those with low-normal, moderately impaired, and severely impaired profiles. The percentage enrolled in a special class increased from 3\% among those with the normal profile to 49 and 93\% among those with the moderately and severely impaired profiles.

For children with $Z_{\mathrm{IQ}}$ between -2 and -1 , the percentage with an IEP was greater for those with a moderately impaired profile based on IQ and EF (83\%) than for those with a lownormal profile $(65 \%$, Table 5$)$. For those with $Z_{\mathrm{IQ}}-2$ or below, the percentage in a special class was greater for those with a severely impaired profile on IQ and EF (93\%) than for those with a moderately impaired profile (60\%).

\section{Discussion}

Using latent profile analysis to summarize nine measures of cognitive ability, we assigned 873 children into four distinct cognitive function subgroups. Three quarters of EP children had normal profiles (34\% normal, 41\% low-normal), whereas $17 \%$ had moderately impaired profiles and $8 \%$ had severely impaired profiles. As hypothesized, the percentage of children in the moderately and severely impaired groups increased with decreasing gestational age at birth. Lending further support to the construct validity of these cognitive profiles, those with more impaired profiles scored lower on basic literacy and math skills, and were more likely to have an IEP, repeat a grade, or be in a separate special class or program. Beyond these findings, the LPA results indicate that some EP children are globally impaired across cognitive domains, whereas others have more specific impairment in executive function. EP children with the low-normal cognitive function profile were much more likely to show impairment in executive function than general reasoning ability, or IQ. However, EP children with the moderately or severely impaired profiles generally showed more global impairment in both IQ and executive function.

Characterizing cognitive function using measures of executive function in addition to IQ better discriminates the academic performance and educational needs of EP children. Given a child's IQ classification, children with more impaired profiles based on IQ and EF had lower academic performance and greater educational needs.

Our findings in EP children (born from 23 to 27 weeks gestation) agree in some respects, and disagree in others, with a study using LPA to classify moderately preterm children (born from 32 to 36 weeks gestation) by cognitive function at the age of 7 years (25). Although our study used assessments of IQ, attention, and executive control that were different from those used by Cserjesi et al., both studies identified four profiles of cognitive functioning, and similar percentages of children falling into the highest functioning to lowest functioning profiles. However, children in the moderately preterm study were of generally higher cognitive ability than children in our EP sample. In particular, among moderately preterm children, mean IQ and attention scores in the two highest profiles were approximately half an SD above the norm and at the norm, respectively, whereas the two highest profiles for EP children corresponded to the performance at the norm and roughly half an SD below the norm, respectively. The lowest functioning profile in the moderately preterm sample had means 1 to 2 SDs below the norm, whereas the lowest functioning profile in the ELGAN sample had means 3 or more SDs below the norm. These differences are consistent with prior findings (and findings from our EP sample) that severity of cognitive impairment increases with decreasing gestational age at birth $(6,15,26)$.

Although the percentage of children with the low-normal profile remained fairly constant for children born from 23 to 27 weeks' gestation, the percentage of children in the moderately and severely impaired groups increased as gestational age at delivery decreased, with an overall prevalence of $25 \%$. In contrast, when we previously examined gestational age effects on IQ in the same cohort (26), impairment was inversely proportional to gestational age, but only $16 \%$ of children were categorized as moderate to severely impaired (two or more SDs below the normative mean) based on measures of IQ. Accordingly, we argue that 


\section{Cognitive functioning in EP children $\quad$ Articles}

IQ does not capture the full impact of cognitive function on outcome, and that the ability of LPA to summarize performance across a broader range of measures improves the ability to identify children at highest risk $(8,9)$.

A strength of this study is that it is based on a large sample of preterm infants identified at birth, with measures of cognitive functioning that include verbal and nonverbal IQ, working memory, attention, set-shifting, inhibition, and mental flexibility. However, a limitation of our study is that other neurocognitive domains including visuospatial processing, language, and memory are not included in our analyses. We do not know how impairment on these domains might have altered the number of profiles that might be identified by an LPA. In addition, our LPA and the identified profiles are based on cognitive functioning scores, rather than on clinically defined impairment. Advantages to this approach are that it allows differentiation of profiles that represent lower but not impaired cognitive functioning, and that the identification of profiles does not depend on cutoff scores for impairment. A limitation is that our groups are not based on clinically defined impairment, although the associations that we found between school achievement and cognitive function determined by the LPA suggest that the results have clinical relevance.

We found that IQ alone does not fully capture cognitive function, and that considering measures of executive function improves the ability to identify impairment in EP children. Overall, 25\% of 10-year-olds born EP had moderate-to-severe impairment based on measures of IQ and EF. Among EP children who were less impaired, there was some evidence of differential impairment specifically in inhibitory control. Our classification of impairment showed the expected associations with gestational age, educational history, and academic achievement.

STATEMENT OF FINANCIAL SUPPORT: This study was supported by a cooperative agreement with the National Institute of Neurological Disorders and Stroke (grants 5U01NS040069-05 and 2R01NS040069-09) and the National Institute of Child Health and Human Development (5P30HD018655-28).

Disclosure: The authors declare no conflict of interest.

\section{REFERENCES}

1. Stoll BJ, Hansen NI, Bell EF, et al. Trends in care practices, morbidity, and mortality of extremely preterm neonates, 1993-2012. J Am Med Assoc 2015;314:1039-51.

2. Anderson PJ. Neuropsychological outcomes of children born very preterm. Semin Fetal Neonat Med 2014;19:90-6.

3. Anderson PJ, Doyle LW. The Victorian Infant Collaborative Group. Neurobehavioral outcomes of school-age children born extremely low birth weight or very preterm in the 1990s. J Am Med Assoc 2003;289: 3264-72.

4. Chyi LJ, Lee HC, Hintz SR, Gould JB, Sutcliffe TL. School outcomes of late preterm infants: special needs and challenges for infants born at 32 to 36 weeks gestation. J Pediatr 2008;153:25-31.
5. Moreira RS, Magalhaes LC, Alves CRL. Effect of preterm birth on motor development, behavior, and school performane of school-age children: a systematic review. J Pediatr 2014;90:119.

6. Johnson S, Fawke J, Hennessy E, et al. Neurodevelopmnetal disability through 11 years of age in children born before 26 weeks of gestation. Pediatrics 2009;124:e249-57.

7. Luu TM, Ment L, Allan W, Schneider K, Vohr BR. Executive and memory function in adolescents born very preterm. Pediatrics 2011;127: e639.

8. Johnson S, Strauss V, Gilmore C, Jaekel J, Marlow N, Wolke D. Learning disabilities among extremely preterm children without neurosensory impairment: comorbidity, neuropsychological profiles and scholastic outcomes. Early Hum Dev 2016;103:69-75.

9. Loe IM, Lee ES, Luna B, Feldman HM. Executive function skills are associated with reading and parent-rated child function in children born prematurely. Early Hum Dev 2012;88:111-8.

10. Schmidt B, Anderson PJ, Doyle LW, et al. Survival without disability to age 5 years after neonatal caffeine therapy for apnea of prematurity. J Am Med Assoc 2012;307:275-82.

11. Shankaran S, Pappas A, McDonald SA, et al. Childhod outcomes after hypothermia for neonatal encephalopathy. N Engl J Med 2012;366:2085-92.

12. Hutchinson EA, De Luca CR, Doyle LW, Roberts G, Anderson PJ. School-age outcomes of extremely preterm or extremely low birth weight children. Pediatrics 2013;131:e1053-61.

13. Taylor HG, Klein N, Drotar D, Schluchter M, Hack M. Consequences and risks of $<1000 \mathrm{~g}$ birth weight for neuropsychological skills, achievement, and adaptive functioning. Dev Behav Pediatr 2006;27:459-69.

14. Linsell L, Malouf R, Morris J, Kurinczuk JJ, Marlow N. Prognostic factors for poor cognitive development in children born very preterm or with very low birth weight: a systematic review. JAMA Pediatr 2015;169: 1162-72.

15. Ritter BC, Nelle M, Steinlin M, Everts R. Influence of gestational age and parental education on executive functions of children born very preterm. J Neonat Biol 2013;2:2167.

16. Leviton A, Kuban KC, O'Shea TM, et al. The relationship between early concentrations of 25 blood proteins and cerebral white matter injury in preterm newborns: the ELGAN study. J Pediatr 2011;158:897-903.

17. O'Shea TM, Allred EN, Dammonn O, et al. The ELGAN study of the brain and related disorders in extremely low gestational age newborns. Early Hum Dev 2009;85:719-25.

18. O'Shea TM, Allred EN, Kuban KC, et al. Elevated concentrations of inflammation-related proteins in postnatal blood predict severe developmental delay at 2 years of age in extremely preterm infants. J Pedicatr 2012;160:395-401.

19. Muthen LK, Muthen BO. MPlus User's Guide, 7th edn. Los Angeles, CA: Muthen \& Muthen, 1998-2012.

20. Schwartz GE. Estimating the dimensions of a model. Ann Stat 1978;6:461.

21. Sclove SL. Application of model-selection criteria to some problems in multivariate analysis. Psychometrika 1987;52:333.

22. Lo $\mathrm{Y}$, Mendell N, Rubin D. Testing the number of components in a normal mixture. Biometrika 2001;88:767.

23. Ramaswamy V, DeSarbo WS, Reibstein DJ, Robinson WT. An empirical pooling approach for estimating marketing mix elasticities with PIMS data. Market Sci 1993;12:103.

24. Lanza ST, Rhoades BL. Latent class analysis: an alternative perspective on subgroup analysis in prevention and treatment. Prev Sci 2013;14:157.

25. Cserjesi R, Van Braeckel KN, Timmerman M, et al. Patterns of functioning and predictive factors in children born moderately preterm or at term. Dev Med Child Neurol 2012;54:710-5.

26. Joseph RM, O'Shea TM, Allred EN, et al. Neurocognitive and academic outcomes at age 10 years of extremely preterm newborns. Pediatrics 2016: 137 . 